\title{
OPTIMIZED FEEDHORN DESIGNS FOR COMPACT ANTENNA TEST RANGE AT LIMOGES UNIVERSITY
}

\author{
Jorge Teniente $^{(1)}$, Eric Arnaud ${ }^{(2)}$, Ramón Gonzalo ${ }^{(1)}$, Carlos del-Río ${ }^{(1)}$ \\ (I) Electric and Electronic Engineering Department. Public University of Navarra. \\ Campus de Arrosadía. E-31006 Pamplona (Navarra) Spain \\ jorge.teniente@unavarra.es,ramon@unavarra.es,carlos@unavarra.es \\ (2) XLIM-OSA - UMR CNRS n 6172. \\ 123, avenue Albert Thomas. 87060 Limoges Cedex, France \\ eric.arnaud@unilim.fr
}

\begin{abstract}
In this paper, high technology optimized feedhorn designs are presented. These feedhorns have been designed to improve the quite zone size and behaviour at the Compact Antenna Test Range (CATR) of the Antenna Research Team (OSA) from Limoges University XLIM laboratory. Very nice results are obtained maintaining the quite zone at its maximum size over the whole frequency range. The designed feedhorns present low crosspolar levels, high phase centre stability and they are very simple and small, resulting in a very low manufacturing cost.
\end{abstract}

\section{INTRODUCTION}

The most simple and cheap indoor antenna measurement chambers are the far field ranges. In fact, these test facilities are the most common solutions in research centres where the cost must be reduced. But, in far field ranges there are two main disadvantages.

At low frequencies (below $1 \mathrm{GHz}$ ), the size of the test antennas is usually a problem to fit inside the test range and the reflectivity of the absorbent material increases, being necessary to increase the size of their pyramids. Then, the total price of the facility increases because it is needed a bigger facility and then more surface to cover with a more expensive absorbent material. This disadvantage is solved usually designing an outdoor antenna measurement facility between two high buildings, between two hills...

At high frequencies (above $30 \mathrm{GHz}$ ), if the gain of the Antenna Under Test (AUT) is high, it is difficult to accomplish with the far field condition:

$$
R=2 D^{2} / \lambda
$$

where $\mathrm{D}$ is the greater dimension of the AUT, $\lambda$ the wavelength and R the distance between the AUT and the feed. A brief calculation demonstrates the great dimension needed to measure the over $30 \mathrm{~dB}$ gain antennas with a usual anechoic chamber above $30 \mathrm{GHz}$. To overcome these difficulties we can require the use of a Compact Antenna Test Range (CATR), see Fig. 1.

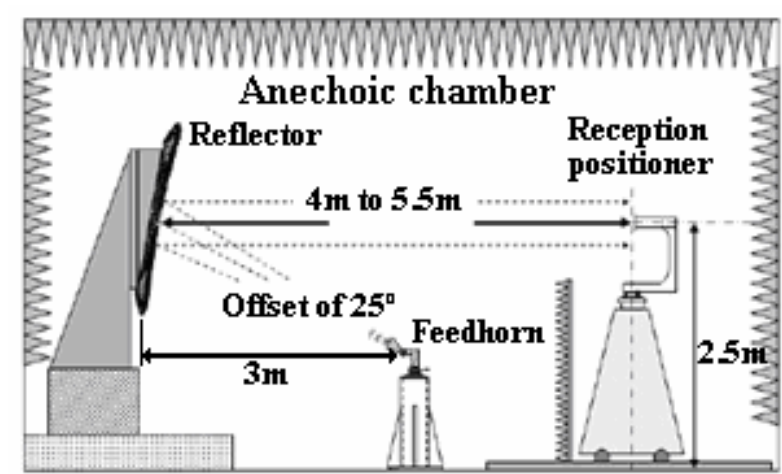

Figure 1. Compact Antenna Test Range (CATR) model at Limoges University

\section{COMPACT ANTENNA TEST RANGE CHARACTERISATION}

A CATR produces a far field condition at a relatively short distance from the feeds, [1, 2]. So, a spherical wave coming from a feed is transformed into a plane wave, after the reflection in a combination of one to three reflectors, depending on the model of the facility. In order that the CATR works properly, this "plane wave region" must meet strict criteria, as a maximum ripple of $0.5 \mathrm{~dB}$ and a phase shift lower than $5^{\circ}$ in the planes parallel to wave front. The volume where these criteria are satisfied is called the Quiet Zone (QZ).

It is of interest that the volume of the QZ to be as big as possible because a bigger volume allows the measurement of bigger antennas. Such volume is limited by the size and the configuration of the mirrors, but the main limit comes usually from the feedhorns used to illuminate the mirror configuration because usually their radiation pattern changes with frequency and the reflectors are not correctly illuminated with big spillover at the lower frequency band of each feedhorn and low illumination efficiencies at the upper frequency band of each of them.

Then, a stabilised radiation pattern feedhorn design would be desirable for any CATR reflector illumination. 


\section{COMPACT ANTENNA TEST RANGE AT LIMOGES UNIVERSITY}

The Limoges' University CATR is composed by a $0.9 \mathrm{~m}$ side square parabolic reflector with a vertical offset whose focal length is 3 metres, see Figs. 1 and 2. The total reflector size is $1.7 \times 1.7$ metres because of rolled edges in order to reduce diffractions [3].

The QZ size is the most important parameter to characterize a CATR. The theoretical size for the QZ at the CATR of Limoges University using a traditional feedhorn is given in Tab. 1 for a maximum ripple of $0.5 \mathrm{~dB}$ and a phase shift lower than $5^{\circ}$.

\section{Table 1. Theoretical values of the QZ size at Limoges' University CATR for a traditional set of feeds}

\begin{tabular}{|c|c|}
\hline Frequency $(\mathrm{GHz})$ & Ripple $<0.5 \mathrm{~dB}$, Phase shift $<5^{\circ}$ \\
\hline $8 \mathrm{GHz}-12 \mathrm{GHz}$ & $40 \mathrm{~cm}<\mathrm{QZ}<107 \mathrm{~cm}$ \\
\hline $12 \mathrm{GHz}-18 \mathrm{GHz}$ & $32 \mathrm{~cm}<\mathrm{QZ}<109 \mathrm{~cm}$ \\
\hline $18 \mathrm{GHz}-26 \mathrm{GHz}$ & $36 \mathrm{~cm}<\mathrm{QZ}<104 \mathrm{~cm}$ \\
\hline $26 \mathrm{GHz}-40 \mathrm{GHz}$ & $32 \mathrm{~cm}<\mathrm{QZ}<108 \mathrm{~cm}$ \\
\hline $33 \mathrm{GHz}-50 \mathrm{GHz}$ & $36 \mathrm{~cm}<\mathrm{QZ}<100 \mathrm{~cm}$ \\
\hline
\end{tabular}

From the values of Tab. 1, it can be seen that the QZ size changes a lot. This effect is due to that a traditional feed presents a radiation pattern that changes a lot with frequency.

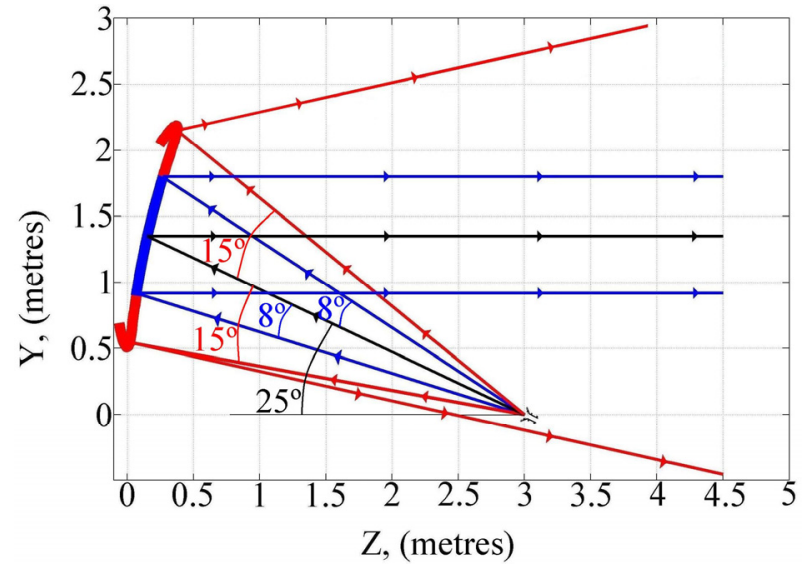

Figure 2. Rolled edge parabolic reflector at CATR

\section{OPTIMIZED FEEDHORN DESIGN}

Traditionally, as feedhorns for CATR test facilities, horns with horizontal corrugations (usually known as chokes or ring loaded slots) have been chosen. These feedhorns have been traditionally used because they present a circular beamwidth, low crosspolarisation levels and because a very directional radiation pattern is not usually required.

The feedhorn design method is accomplished optimizing the radiation pattern response of the feeds to the optimum radiation pattern given by the reflector designer, and then discretizing the whole frequency band needed in the test facility in; as less feedhorns as possible.
The problem arises in the derivation obtained for the radiation pattern of each feedhorn at the frequency band borders. This derivation produces a low efficiency illumination of the reflector and the QZ size is strongly affected as it can be seen in Tab. 1 .

This problem can be solved by means of a stabilised radiation pattern feedhorn design with enough bandwidth, (a feedhorn with compensated gain in the whole frequency band).

To design such a feedhorn, vertical corrugations should be included in the design. In fact for the design we have used an internationally patented feedhorn prototype that combines in the same profile both horizontal and vertical corrugations. This patent belongs to the Public University of Navarra in Spain, [4, 5].

We have also used for the analysis and optimization of the profile a very nice commercial code called Mician $\mu$ Wave Wizard (http://www.mician.com).

The specifications to be achieved in the design have been given by the reflector designer. These specifications can be found in Tab. 2 .

Table 2. Optimum specifications for the feedhorn Limoges' University CATR design

\begin{tabular}{|c|c|c|c|c|c|}
\hline & Feed & Feed & Feed & Feed & Feed \\
& 1 & 2 & 3 & 4 & 5 \\
\hline Bandwidth, (GHz) & $8-$ & $12-$ & $18-$ & $26-$ & $33-$ \\
& 12 & 18 & 26 & 40 & 50 \\
\hline Beamwidth, $-0.5 \mathrm{~dB}$ & $\pm 8^{\circ}$ & $\pm 8^{\circ}$ & $\pm 8^{\circ}$ & $\pm 8^{\circ}$ & $\pm 8^{\circ}$ \\
\hline Beamwidth, $-3 \mathrm{~dB}$ & $\pm 15^{\circ}$ & $\pm 15^{\circ}$ & $\pm 15^{\circ}$ & $\pm 15^{\circ}$ & $\pm 15^{\circ}$ \\
\hline VSWR & $<1.2$ & $<1.2$ & $<1.2$ & $<1.2$ & $<1.2$ \\
\hline $\begin{array}{c}\text { Crosspolarisat., } \\
\text { (dB) }\end{array}$ & $<-25$ & $<-25$ & $<-25$ & $<-25$ & $<-25$ \\
\hline
\end{tabular}

From the specifications it can be observed that the total bandwidth of the feedhorns is in between $36.3 \%$ for feed 3 to $42.4 \%$ for feed 4 . So feed 4 , will fix the needed bandwidth of the design.

A design with horizontal and vertical corrugations was prepared for the Mician $\mu$ Wave Wizard software. Such initial design was optimized with the own software's optimization tool and the obtained results were really nice.

\section{SIMULATED RESULTS}

After the optimization process, a set of scaled versions for the five feedhorns was obtained with a total size of $1.5 \cdot \lambda$ length and $3.1 \cdot \lambda$ width. The resultant optimised design is a profile with a taper from monomode waveguide, one horizontal corrugation and three vertical corrugations, see Fig. 3.

Each of the feedhorns has the same scaled profile to cover the different frequency bands, because their design specifications were the same. In Fig. 3 a size comparison of the biggest versus the smallest one can be seen and compared. 


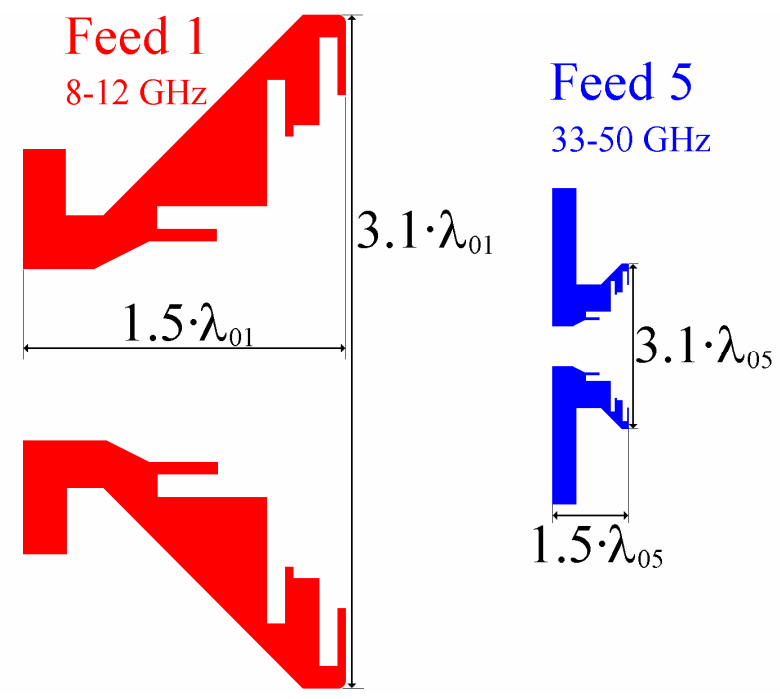

Figure 3. Designed feedhorn profiles 1 and 5

Hay et al. [6] has shown that a perfectly constructed special purpose corrugated horn, when measured in a very high performance anechoic chamber, gives exact agreement with theoretical patterns with crosspolar maximum $<-50 \mathrm{~dB}$. So, simulated results of the following lines will be very near the real measured ones although we can only present at the moment a few measured results.

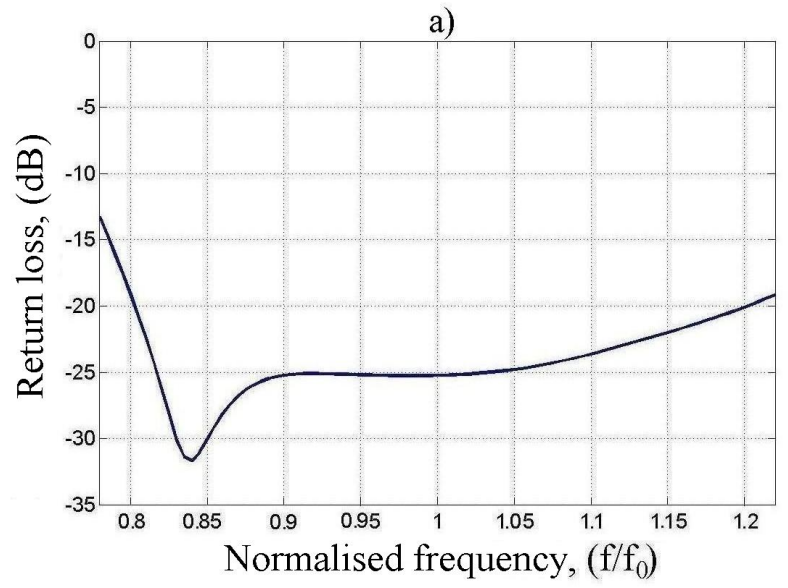

b)

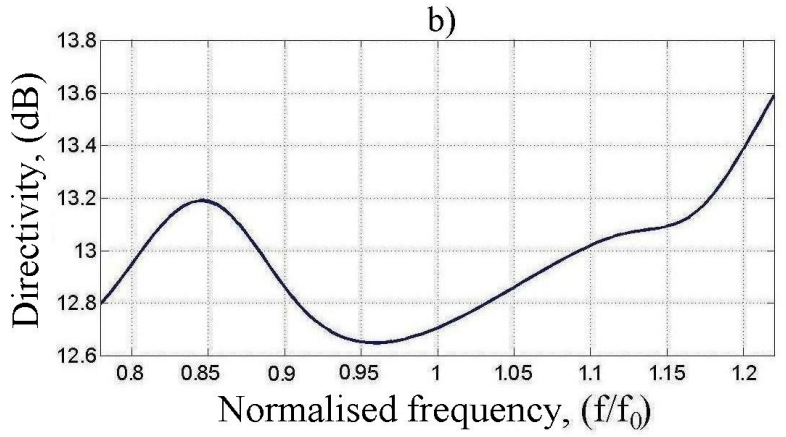

Figure 4. a) Simulated return loss b) Simulated directivity
Return loss results meet fairly well specifications, the simulated results shows a worst case of $-18 \mathrm{~dB}$ of return loss (1.3 of VSWR) at lower band of each feedhorn, see Fig. 4a. VSWR response is below 1.2 in a $40 \%$ bandwidth.

Fig. 4b shows the directivity variation in the whole band. The directivity change is very small, (from 12.6 to $13.4 \mathrm{~dB}$ in more than a $40 \%$ bandwidth). This result will produce a nice stability in the radiation pattern beamwidth. The QZ size will not change too much with frequency as we will demonstrate in the following lines. Simulated crosspolarisation requirements are met by far, see Fig. 5a. The worst value is $-27 \mathrm{~dB}$ and the reflector including the rolled edges ( \pm 15 degrees) intercepts crosspolar levels well below $-34 \mathrm{~dB}$, the parabolic reflector $( \pm 8$ degrees $)$ will intercept even less crosspolarised component.

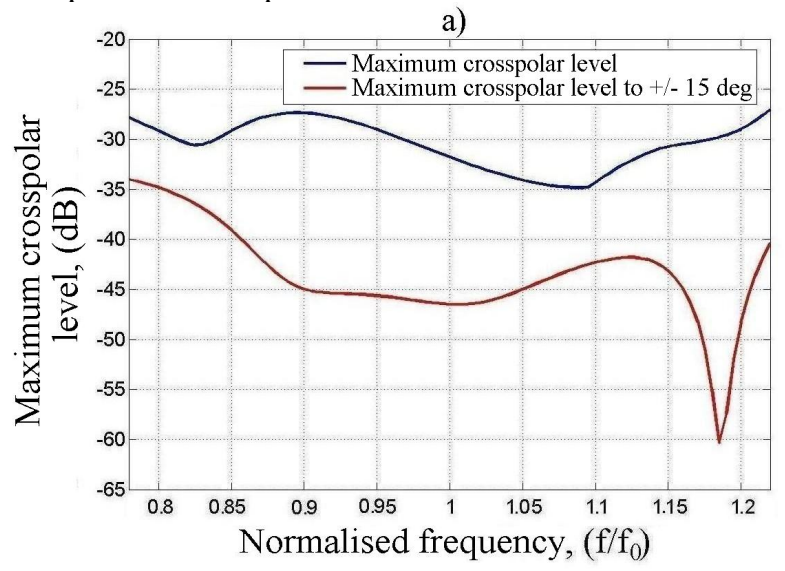

b)

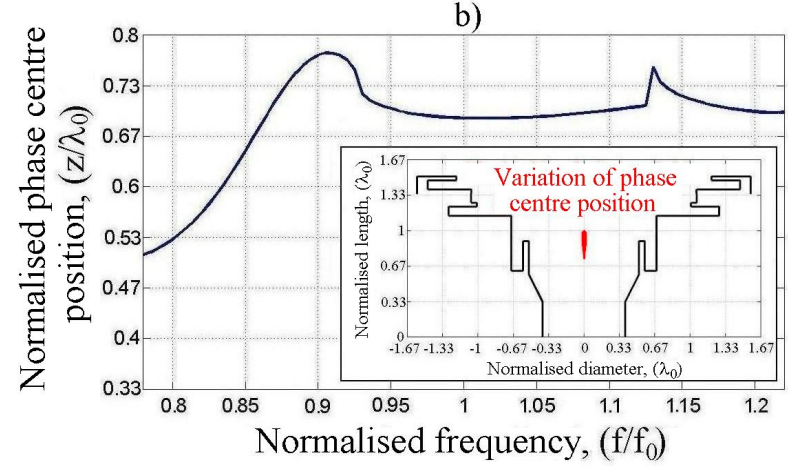

Figure 5. a) Simulated maximum crosspolar level

b) Simulated relative phase centre position and its position inside the antenna (values are referred to the aperture of the feedhorn)

On the other hand, it is important to remark the simulated stability of the phase centre position, see Fig. 5b. This parameter is very important for feeds being used to illuminate reflectors because the feed can be placed in the focus of the reflector more accurately in the whole frequency band. For this set of feedhorns, the maximum variation of the phase centre is of $\pm \lambda_{0} / 8$, then 
$\pm 3.75 \mathrm{~mm}$ for feed 1 and $\pm 0.9 \mathrm{~mm}$ for feed 5 . It is also observed in Fig. $5 \mathrm{~b}$ that in more than a $35 \%$ of the whole frequency band, the phase centre variation is even less, about $\pm \lambda_{0} / 24$.

Simulated results for illumination angles of 8 degrees and 15 degrees are presented in Fig. 6. The requirements were an illumination of $-0.5 \mathrm{~dB}$ at 8 degrees and $-3 \mathrm{~dB}$ at 15 degrees, see Tab. 2 .

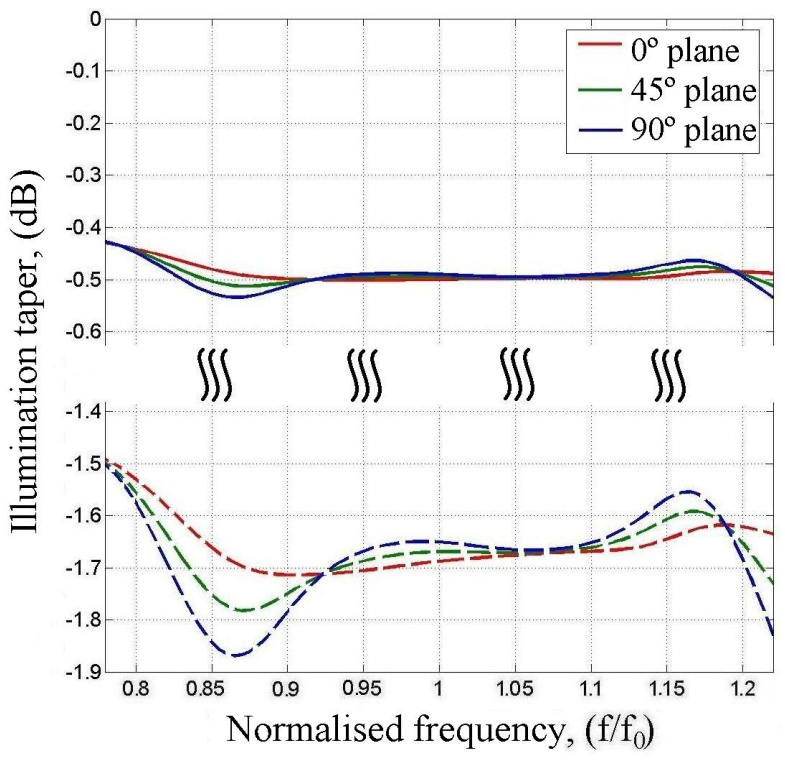

Figure 6. Simulated illumination taper of feedhorns at $8^{\circ}$ (solid lines) and at $15^{\circ}$ (dashed lines)

At 8 degrees the requirement is nicely met, in fact the illumination at 8 degrees is the most important parameter in the feedhorn design because this is the angle that intercepts the parabolic reflector an determines then the size of the quite zone.

On the other hand, at 15 degrees the obtained illumination is bigger than the requirement $(-3 \mathrm{~dB})$. An obtained illumination around $-1.6 \mathrm{~dB}$ is obtained at 15 degrees. This parameter is difficult to reduce with the designed profile without affecting the bandwidth. In fact we consider at the moment that, there is no possible feedhorn design that could lower this 15 degrees illumination maintaining the rest of the specified parameters with such a nice behaviour in a $40 \%$ bandwidth. This illumination is not so important than the 8 degrees one because the rolled edges of the reflector will reduce this radiation in the QZ, see Fig. 2.

Table 3. Theoretical values of the $Q Z$ size at the CATR of Limoges University for the new set of feeds

\begin{tabular}{|c|c|}
\hline Normalised frequency, $\left(\mathrm{f} / \mathrm{f}_{0}\right)$ & $0.8-1.2$ \\
\hline $\begin{array}{c}\text { Ripple }<0.5 \mathrm{~dB} \\
\text { Phase shift }<5^{\circ}\end{array}$ & $86 \mathrm{~cm}<\mathrm{QZ}<90 \mathrm{~cm}$ \\
\hline $\begin{array}{c}\text { Ripple }<1 \mathrm{~dB} \\
\text { Phase shift }<10^{\circ}\end{array}$ & $107 \mathrm{~cm}<\mathrm{QZ}<109 \mathrm{~cm}$ \\
\hline
\end{tabular}

In fact, in Tab. 3 we can conclude easily that the QZ is much more stable in the whole frequency band with the new set of feedhorns if we compare it with Tab. 1 .

The final size of the QZ with the new set of feedhorns will be of $86 \mathrm{~cm}$. This size will allow the measurement in the Limoges' University CATR, with the best precision in the worst case, of a parabolic reflector of $35 \mathrm{~dB}$ gain at $8 \mathrm{GHz}$ and a $50 \mathrm{~dB}$ gain one at $50 \mathrm{GHz}$.

In Fig. 7, simulations at several frequencies of the set of feedhorns are presented. In Fig. 8, feedhorn 5 manufactured model that will work from 33 to $50 \mathrm{GHz}$ is being prepared to be used in the CATR.
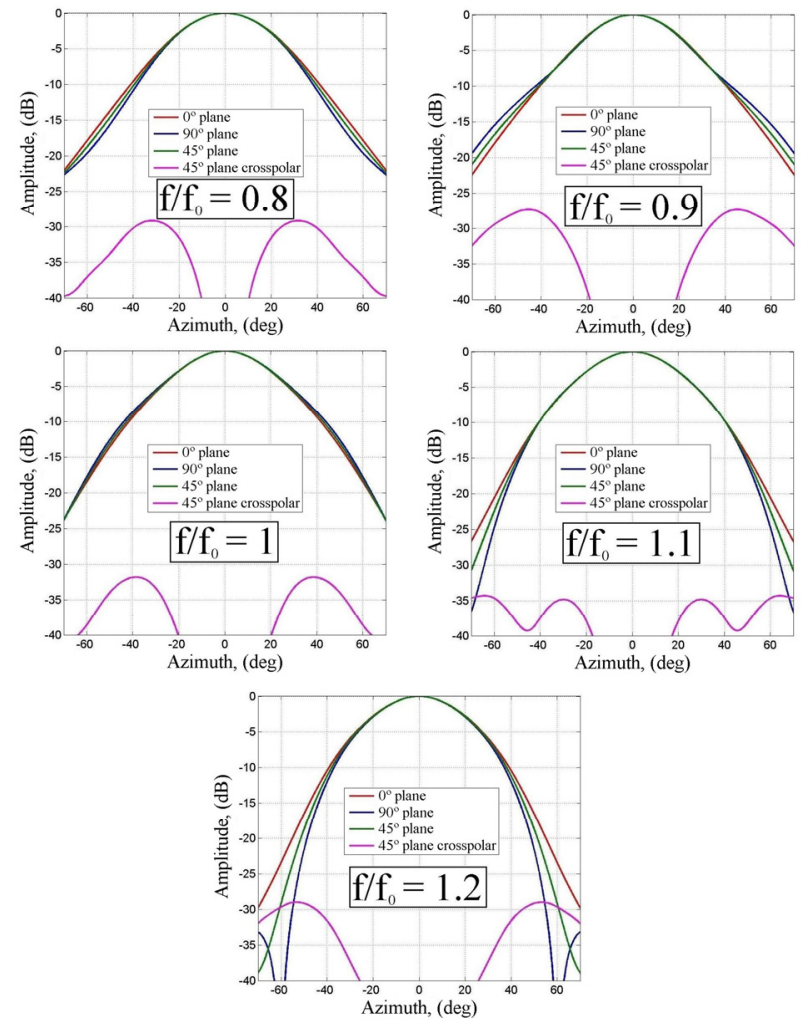

Figure 7. Simulated radiation patterns

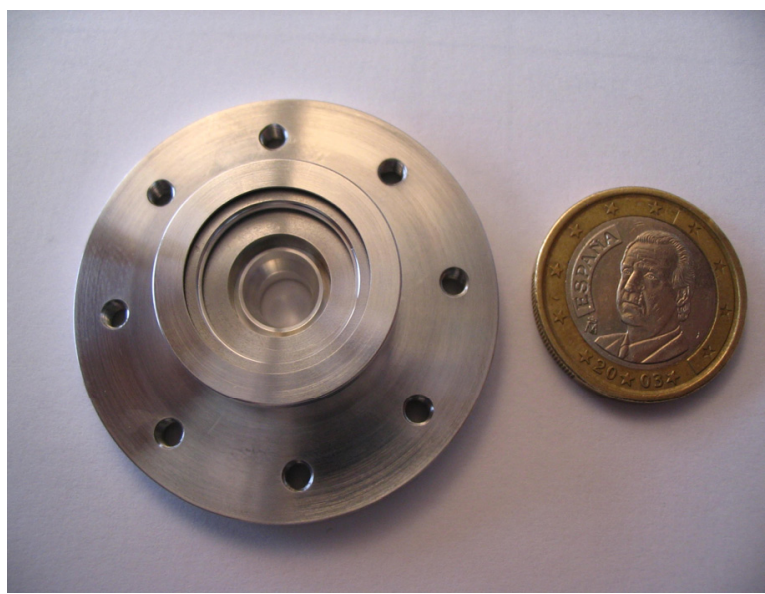

Figure 8. Photograph of the manufactured feedhorn 5 (33-50 GHz).

A $1 €$ coin is photographed to compare 


\section{MEASURED RESULTS}

At the paper's absolute deadline for submission, we have only measured results for the smallest prototype, feedhorn 5, see Fig. 8. Measured results agree really nice with simulated ones, (no crosspolar measured data available yet). See Fig. 9 for a comparison between simulated and measured patterns for feedhorn 5 .
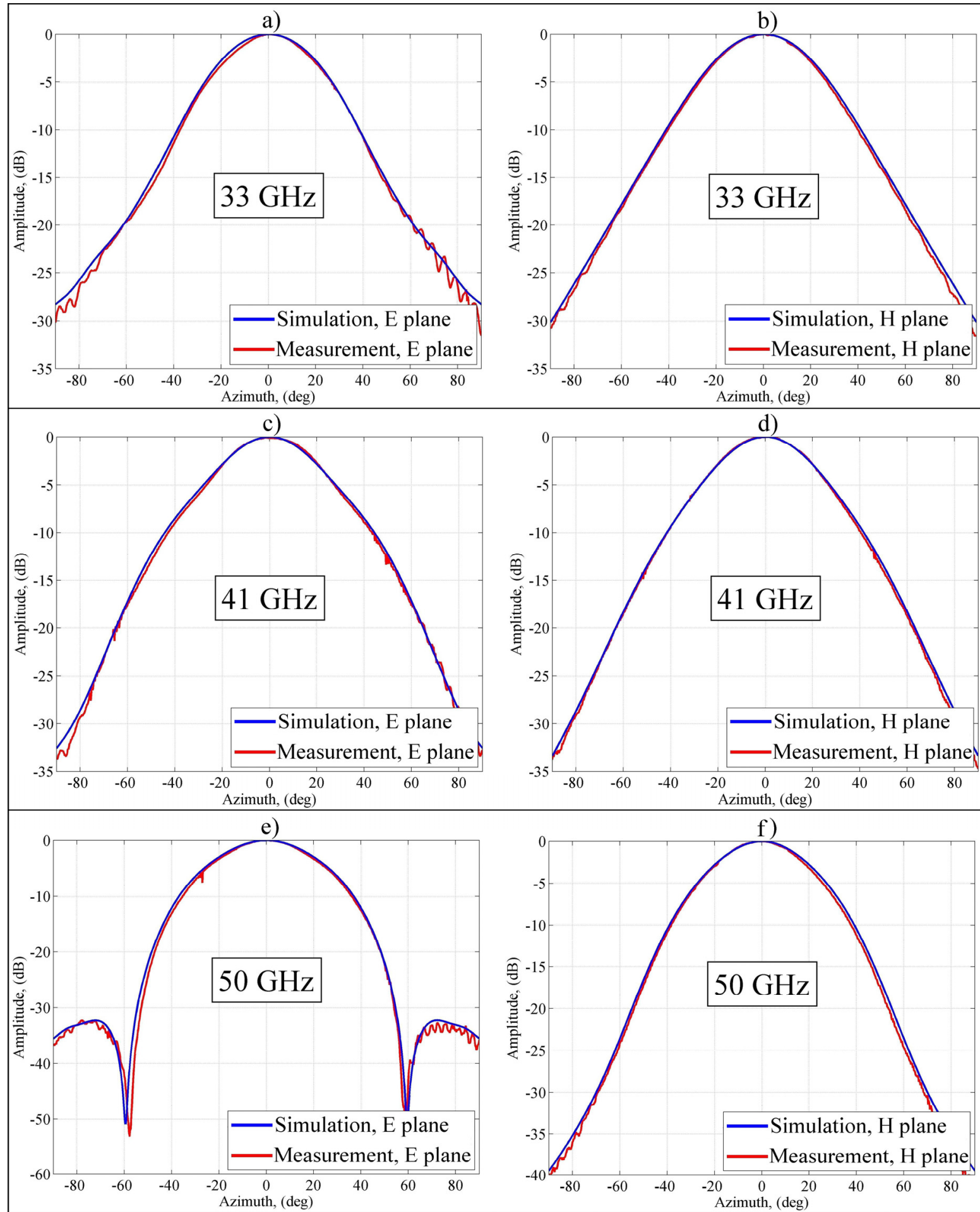

Figure 9. Comparison between simulated and measured radiation patterns for feedhorn 5
a) E plane at $33 \mathrm{GHz}$
b) $\mathrm{H}$ plane at $33 \mathrm{GHz}$
c) E plane at $41 \mathrm{GHz}$
d) $\mathrm{H}$ plane at $41 \mathrm{GHz}$
e) E plane at $50 \mathrm{GHz}$
f) H plane at $50 \mathrm{GHz}$ 


\section{CONCLUSIONS AND FUTURE RESEARCH}

A set of optimised feedhorns for the Limoges University CATR has been designed and manufactured. The prototypes are being measured.

The simulated results show a nice stability of the QZ over the whole bandwidth $(8-50 \mathrm{GHz})$ and a maximization of its size.

The designed set of feedhorns is very simple, small, easy to manufacture and presents nice phase centre stability in the whole band. As corrugated horns, manufacture accuracy is not so important.

A measurement campaign is being performed at present, the results shown for feedhorn 5 agree very well with simulated ones.

Simulations in three dimensions employing accurate methods as Physical Optics (PO) and Physical Theory of Diffraction (PTD) are intended to be made to fully characterize the QZ with this set of feedhorns.

\section{REFERENCES}

1. Olver A.D., Compact Antenna Test Ranges, Antennas and Propagation, 1991. ICAP91, Seventh International Conference, pages 99-108, vol. 1

2. Huff J.D. and. Brechin C. B., A Millimeter Compact Range, IEE Colloquium on Antenna Measurements using the Compact Antenna Test Range. 1991. p. $9 / 1-9 / 8$

3. Arnaud E., Belot D., Jecko B. and Thevenot M., Design, realisation and characterisation of a low cost compact antenna test range, 11th International Symposium on Antenna Technology and Applied Electromagnetics. ANTEM 2005. Saint Malo, France. June 2005

4. R. Gonzalo, C. del Río, D. Goñi, J. Teniente, Horn antenna combining horizontal and vertical ridges, International patent number: WO03/100907. Priority country: Spain. Priority date: 24th May 2002. Property of: Public University of Navarra. PCT extension date: $17^{\text {th }}$ May 2003. International publication date: $4^{\text {th }}$ December 2003 .

5. Teniente J., Gonzalo R. and del-Río C., Choked Gaussian Antenna: Extremely low Sidelobe Compact Antenna Design, IEEE Antennas and Wireless Propagation Letters, Vol. 1, 2002

6. Hay S. G., Barker S. J., Granet C., Forsyth A. R., Bird T. S., Sprey M. A. and K. J. Greene, Earth station antenna for an European teleport application, IEEE Antennas and Propagation Society International Symposium, No. 2, pp. 300303, 2001. 